\title{
ESBL-producing $\mathrm{E}$. coli and $\mathrm{K}$. pneumoniae: prevalence and antimicrobial susceptibility
}

\author{
Giuseppe Miragliotta², Anna Di Taranto', Rossella De Nittis', Raffaele Antonetti ${ }^{1}$, \\ Raffaele Del Prete', Adriana Mosca² \\ ${ }^{1}$ Laboratorio di Microbiologia, Ospedali Riuniti di Foggia \\ ${ }^{2}$ Sezione di M icrobiologia, Dip. M IDIM , Università di Bari
}

Key Words: E. coli, K. pneumoniae, ESBL, sensibilità antibiotica

\section{Valutazione dell'antibiotico resistenza in ceppi di E. coli e K. pneumoniae produttori di ESBL}

\section{SUMMARY}

Infections caused by extended-spectrum beta-lactamase (ESBL) producing E. coli and K. pneumoniae limit therapeutical options. The objective of this study was to evaluate the activity of ciprofloxacin, imipenem, amikacin and piperacillin/tazobactam against ESBL-producing E. coli and K. pneumoniae strains isolated from patients admitted to $O$ spedali Riuniti, Foggia, over the period January-D ecember 2007. A total of $1013 \mathrm{E}$. coli and $146 \mathrm{~K}$. pneumoniae were isolated from various clinical specimens. 111/1013 (10.9\%) and 24/146 (16.4\%) were confirmed as ESBL-producing strains by both the Phoenix system (Becton \& Dickinson) and the E-test (bioMérieux, Italia). Both the ESBL producing enterobacteria showed a high degree resistance to ciprofloxacin; in addition, K. pneumoniae exhibited resistance also to piperacillin/tazo bactam. Imipenem was the most effective antibiotic with the exception of one strain ESBL-positive K. pneumoniae. A similar pattern was showed by amikacin. In view of the increasing prevalence of ESBL strains it appears important a continuous antimicrobial monitoring.

Received April 17, 2009

Accepted June 26, 2009

\section{INTRODUZIONE}

Escherichia coli e Klebsiella pneumoniae sono enterobatteri produttori di beta lattamasi a spettro esteso (ESBL) e rappresentano un problema di notevole rilievo clinico ed epidemiologico (7). Le ESBL sono enzimi in grado di idrolizzare una vasta gamma di antibiotici beta-lattamici inclusi penicillina, cefalosporine a spettro ristretto ed esteso, monobattamici e sono normalmente inibite dagli inibitori delle ß-lattamasi come l'acido clavulanico. Oltre alle ben note ESBL di derivazione TEM e SHV, oggi si conoscono numerosi enzimi idrolitici a spettro esteso che hanno origine da geni importati da altri microrganismi (CTX-M) (2).

ESBL sono state descritte per la prima volta in Germania nel 1983, successivamente sono state segnalate in tutto il mondo e la loro frequenza è in costante aumento raggiungendo in alcune aree geografiche livelli prossimi al 50\% (1). I processi infettivi da enterobatteri ESBL produttori sono prevalenti in ambiente ospedaliero $(5,3)$ e spesso complicano il decorso clinico di pazienti affetti da polipatologie. Diversi studi policentrici nazionali indicano che la percentuale di isolamento di enterobatteri ESBL positivi oscilla tra il 4 e il $12 \%$ in base all'area geografica e alla specie (11 ). Un aspetto preoccupante, recentemente emerso, è l'isolamento di questi enzimi (soprattutto del tipo CTX-M) in ambito comunitario (8) in pazienti che presentano alcuni fattori di rischio quali: età avanzata, diabete, infezioni urinarie ripetute, uso di chinoloni o almeno una ospedalizzazione nel corso dell'anno precedente (9).

Alcuni ceppi ESBL-produttori presentano il fenomeno della multiresistenza nei confronti di fluorochinoloni, cotrimossazolo e aminoglicosidi riducendo drasticamente le opzioni terapeutiche a disposizione del clinico (12). L'utilizzo dei carbapenemi ha determinato l'insorgenza di ceppi ESBL-positivi resistenti (4). La presenza di ESBL, dunque, prevale in ceppi ospedalieri, determina multi-resistenza, rischio di diffusione epidemica e per questo necessita di sorveglianza epidemiologica.

Scopo del nostro lavoro è stato valutare la presenza dei ceppi di E. coli e K. pneumoniae ESBL-

\section{Corresponding author: Giuseppe Miragliotta}

Sez microbiologia Dip MIDIM, Università di Bari - Policlinico

Piazza G. Cesare, 70I22 Bari - Tel 0805478504 - Fax 0805478504

E-mail: miragliotta@midim.uniba.it 
produttori e la loro resistenza nei confronti di ciprofloxacina, amikacina, imipenem e piperacillina/tazobactam cui con maggior frequenza si ricorre in caso di sospetta presenza di enterobatteri ESBL-produttori.

\section{MATERIALI E METODI}

Nel periodo Gennaio-Dicembre 2007 presso il Laboratorio di Microbiologia degli Ospedali Riuniti di Foggia sono stati isolati 1013 ceppi di E. coli e 146 ceppi di K. pneumoniae da campioni biologici (soprattutto urine e broncoaspirato) di pazienti ricoverati nei reparti di Rianimazione, Medicina, Lungodegenza, Nefrologia o afferenti all'Ambulatorio dell'Ospedale. I test biochimici e i test di sensibilità sono stati eseguiti con lo strumento Phoenix (Becton \& Dickinson) che permette contemporaneamente di valutare la produzione delle ESBL attraverso l'uso di alcune cefalosporine da sole o in combinazione con acido clavulanico (CA): cefpodoxime, ceftazidime, ceftazidime/CA, cefotaxime/CA e ceftriaxone/CA (10). La produzione di ESBL è stata confermata con il metodo E-test (bioMérieux, Italia), utiliz- zando strisce cefotaxime/ cefotaxime/CA e ceftazidime/ceftazidime/CA.

\section{RISULTATI}

Per quanto riguarda la ricerca delle ESBL, 111/1013 (10.9\%) ceppi di E. coli e 24/146 (16.4\%) ceppi di $K$. pneumoniae sono risultati positivi. In Tabella 1 sono riportate le percentuali di isolamento dei ceppi ESBL in base al reparto di provenienza. Il maggior numero di ceppi di E. coli e K. pneumoniae sono stati isolati dall'ambulatorio, dove però si è osservata la più bassa percentuale di ceppi ESBL (6.1\% e 7.2\% rispettivamente). Il maggior numero di ceppi ESBL positivi di E. coli sono stati isolati dal reparto di Medicina (22.4\%), mentre quelli di K. pneumoniae (66.6\%) sono stati isolati dai reparti di Rianimazione e Lungodegenza. Per quanto riguarda la resistenza agli antibiotici testati i risultati sono riportati in Tabella 2 e 3. Ciprofloxacina ha mostrato la più alta percentuale di resistenza nei confronti sia di E. coli (82.8 \%) che di K. pneumoniae (91.6\%). Imipem è risultato l'antibiotico più attivo fatta eccezione di un solo ceppo di K. pneumoniae isolato dalle urine

Tabella 1. Percentuale di isolamento dei ceppi E. coli e K. pneumoniae ESBL-produttori

\begin{tabular}{lcccc}
\hline REPARTO & \multicolumn{2}{c}{ E.coli } & \multicolumn{2}{c}{ K. pneumoniae } \\
& $\mathbf{N}^{\circ}$ ceppi & ESBL+ (\%) & $\mathbf{N}^{\circ}$ ceppi & ESBL+ (\%) \\
\hline Rianimazione & 15 & $2(13,3 \%)$ & 5 & $4(66,6 \%)$ \\
\hline Medicina & 205 & $46(22,4 \%)$ & 26 & $4(15,3 \%)$ \\
\hline Lungo degenza & 21 & $3(14,3 \%)$ & 3 & $2(66,7 \%)$ \\
\hline Ambulatorio & 672 & $41(6,1 \%)$ & 83 & $5(7,2 \%)$ \\
\hline N efrologia & 100 & $19(19 \%)$ & 29 & $9(31,9 \%)$ \\
\hline TOTALE & $\mathbf{1 0 1 3}$ & $\mathbf{1 1 1}(10,9 \%)$ & $\mathbf{1 4 6}$ & $\mathbf{2 4}(16,4 \%)$ \\
\hline
\end{tabular}

Tabella 2. Resistenze di E. coli ESBL-produttori

\begin{tabular}{|c|c|c|c|c|}
\hline REPARTO ( ${ }^{\circ}$ ceppi) & $\begin{array}{l}\text { AN } \\
\text { AN }\end{array}$ & CIP & IPM & TZP \\
\hline Rianimazione (2) & 0 & 2 & 0 & 0 \\
\hline Medicina (46) & 0 & 40 & 0 & 3 \\
\hline Lungo degenza (3) & 0 & 3 & 0 & 1 \\
\hline Ambulatorio (41) & 0 & 33 & 0 & 4 \\
\hline N efrologia (19) & 0 & 14 & 0 & 2 \\
\hline TOTALE (111) & 0 & 92 & $\mathbf{0}$ & 10 \\
\hline
\end{tabular}

AN , amikacina - CIP, ciprofloxacina - IPM, imipenem - TZP, piperacillina/tazobactam

Tabella 3. Resistenze di K. pneumoniae ESBL-produttori

\begin{tabular}{|c|c|c|c|c|}
\hline REPARTO ( ${ }^{\circ}{ }^{\circ}$ ceppi) & $\begin{array}{l}\text { AN } \\
\text { AN }\end{array}$ & CIP & IPM & TZP \\
\hline Rianimazione (4) & 0 & 4 & 0 & 4 \\
\hline Medicina (4) & 1 & 3 & 0 & 3 \\
\hline Lungo degenza (2) & 1 & 1 & 0 & 1 \\
\hline Ambulatorio (5) & 1 & 6 & 1 & 6 \\
\hline N efrologia (9) & 0 & 8 & 0 & 7 \\
\hline TOTALE (24) & 3 & 22 & 1 & 21 \\
\hline
\end{tabular}

AN , amikacina - CIP, ciprofloxacina - IPM, imipenem - TZP, piperacillina/tazobactam 
di un paziente nefrologico afferente all'ambulatorio. Amikacina e piperacillina/tazobactam sono risultate più attive nei confronti di $E$. coli (percentuale di resistenza rispettivamente 0 e $9 \%$ ) rispetto a K. pneumoniae $(12.5 \%$ e $87.5 \%$ ).

\section{DISCUSSIONE}

Nei paesi industrializzati i patogeni chiamati in causa nelle infezioni ospedaliere sono fondamentalmente gli stessi, sebbene con percentuali di isolamento differenti (3 ). Per quanto riguarda gli enterobatteri ed in particolare E. coli e K. pneumoniae il problema più rilevante è rappresentato dalla produzione di ESBL che conferiscono resistenza a cefalosporine e monobattami. Inoltre, i ceppi ESBL-produttori portano spesso determinanti di resistenza nei confronti di fluorochinoloni, cotrimossazolo e aminoglicosidi (1 ). ESBL hanno attualmente una disseminazione globale, non solo negli ospedali ma anche a livello comunitario e nelle strutture di lungodegenza. Studi di sorveglianza riportano che solamente i carbapenemici sono attivi nei confronti degli Enterobatteri produttori di ESBL, sebbene sia stata segnalata l'emergenza di ceppi resistenti (6). Amikacina presenta resistenza nel 20\% dei casi. La percentuale di resistenza è più elevata per gentamicina (> 50\%) e raggiunge il 70\% per i fluorochinoloni. Questi dati sono in linea con quelli della letteratura internazionale, che riportano i risultati di altre indagini di sorveglianza con l'attività microbiologica dei carbapenemi completa, mentre diminuisce in maniera sensibile quella di amikacina e dei fluorochinoloni (11 ). I nostri dati mostrano che le percentuali di isolamento dei ceppi ESBL di $E$. coli e $K$. pneumoniae sono rispettivamente di $10,6 \%$ e $16,4 \%$ e rientrano nella media nazionale. Per E. coli la produzione di ESBL è sempre associata alla resistenza alla ciprofloxacina (82.8\%). Imipenem ed amikacina sono farmaci sempre attivi ( $100 \%$ di sensibilità) come pure la piperacillina/tazobactam con sensibilità del 91\%. Maggiore è la resistenza dei ceppi ESBL di $K$. pneumoniae che risultano resistenti a ciprofloxacina e piperacillina/tazobactam (91.6\% e 87.5\%). Inoltre, 3/24 (12.5\%) ceppi sono risultati resistenti ad amikacina ed è stato isolato un ceppo resistente anche ad imipenem. In conclusione, nella nostra realtà loco-regionale circolano ceppi di E. coli e $K$. pneumoniae produttori di ESBL con una elevata resistenza alla ciprofloxacina. In aggiunta alla ciprofloxacina i ceppi di K. pneumoniae mostrano resistenza anche alla piperacillina/tazobactam, alcuni all'amikacina ed un ceppo all'imipemen.
Ciò significa che questi ceppi sono in rapida evoluzione in risposta alla pressione selettiva creata dall'uso di questi antibiotici.

E' pertanto fondamentale una sorveglianza epidemiologica capillare e continuativa associata ad una diagnostica di laboratorio corretta e specifica.

\section{BIBLIOGRAFIA}

1. Biedenbach DJ, Moet GJ, Jones RN. Occurrence and antimicrobial resistance pattern comparisons among bloodstream infection isolates from the SENTRY Antimicrobial Surveillance Program (1997-2002). Diagn Microbiol Infect Dis 2004 Sep; 50(1):59-69

2. Brigante G, Luzzaro F, Perilli M, et al. Evolution of CTX-M-type beta-lactamases in isolates of Escherichia coli infecting hospital and community patients. Int J Antimicrob Agents 2005 Feb; 25(2): 157-62

3. Canton R, Novais A, Valverde A, et al. Prevalence and spread of extended-spectrum beta-lactamase-producing Enterobacteriacae in Europe. Clin Microbiol Infect 2008; 14(suppl 1): 144-53

4. Gulmez D, Woodford N, Palepou MF, et al. Carbapenem-resistant Escherichia coli and Klebsiella pneumoniae isolates from Turkey with OXA-48-like carbapenemases and outer membrane protein loss. Int J Antimicrob Agents 2008; 31(6): 523-6

5. Kjerulf A, Hansen DS, Sandvang D, et al. The prevalence of ESBL-producing E. Coli and Klebsiella strains in the Copenhagen area of Denmark. APMIS 2008; 116(2): 118-24

6. Nordmann P, Cuzon G, Naas T. The real threat of Klebsiella pneumoniae carbapenemase-producing bacteria. Lancet Infect Dis 2009, 9:228-36 Pitout JD, Laupland KB. Extended-spectrum beta-lactamaseproducing Enterobacteriacae: an emerging pubblichealth concern. Lancet Infect Dis 2008; 8(3): 159-66

7. Pitout JD, Laupland KB. Extended-spectrum beta-lactamase-producing Enterobacteriacae: an emerging pubblic-health concern. Lancet Infect Dis 2008; 8(3): 159-66

8. Pitout JD, Nordmann $\mathrm{P}$, Laupland $\mathrm{KB}$, et al. Emergence of Enterobacteriaceae producing extended-spectrum beta-lactamases (ESBLs) in the community. J Antimicrob Chemother 2005; 56(1):52-9

9. Rodriguez-Bano J, Navarro MD. Extended-spectrum beta-lactamases in ambulatory care: a clinical perspective. Clin Microbiol Infect 2008, 14:104-10

10. Sanguinetti M, Posteraro B, Spanu $\mathrm{T}$, et al Characterization of clinical isolates of Enterobacteriaceae from Italy by the BD Phoenix extended-spectrum beta-lactamase detection method. J Clin Microbiol 2003; 41(4):1463-8

11. Spanu T, Luzzaro F, Perilli M, et al. Italian ESBL Study Group. Occurrence of extended-spectrum betalactamases in members of the family Enterobacteriaceae in Italy: implications for resistance to beta-lactams and other antimicrobial drugs. Antimicrob Agents Chemother 2002; 46(1):196-202

12. Tamayo J, Orden B, Cacho J, et al. Activity of ertapenem and other antimicrobials against ESBLproducing enterobacteria isolated from urine in patients from Madrid. Rev Esp Quimioter 2007; 20(3): 334-8 